This is the version of the article before peer review or editing, as submitted by an author to Journal of Neural Engineering. IOP Publishing Ltd is not responsible for any errors or omissions in this version of the manuscript or any version derived from it. The Version of Record is available online at doi:10.1088/1741-2552/aae191.

\title{
Multilayer 3D electrodes for neural implants
}

\author{
Marta Jole Ildelfonsa Airaghi Leccardi ${ }^{1}$, Paola Vagni ${ }^{1}$, and Diego Ghezzi ${ }^{1}$ \\ ${ }^{1}$ Medtronic Chair in Neuroengineering, Center for Neuroprosthetics and Institute of Bioengineering, \\ School of Engineering, École Polytechnique Fédérale de Lausanne, Lausanne, Switzerland.
}

E-mail: diego.ghezzi@epfl.ch

\begin{abstract}
Objective. In many applications, multielectrode arrays exploited as neural implants requires a high density and a high number of electrodes to precisely record and stimulate the activity of the nervous system while preserving the overall size of the array as little as possible. Approach. Here we present a multilayer and three-dimensional (3D) electrode array, together with its manufacturing method, enabling a higher electrode density and a more efficient signal transduction with the biological tissue. Main results. The 3D structure of the electrode array allows a multilayer placement of the interconnects within a flexible substrate, it narrows the probe size per the same number of electrodes, and it maintains the electrode contacts at the same level within the tissue. In addition, it increases the electrode surface area, leading to a lower electrochemical impedance and a higher charge storage capacity. To characterize the recordings capabilities of the multilayer $3 \mathrm{D}$ electrodes, we measured visually evoked cortical potentials in mice and analyzed the evolution of the peak prominences and latencies according to different light intensities and recording depths within the brain. The resulting signal-to-noise ratio is improved compared to flat electrodes. Finally, the 3D electrodes have been imaged inside a clarified mouse brain by using a light-sheet microscope to visualize their integrity within the tissue. Significance. The multilayer 3D electrodes have proved to be a valid technology to ensure tissue proximity and higher recording/stimulating efficiencies while allowing an increase in the electrode density and a reduction in the probe size.
\end{abstract}

Keywords: 3D electrode arrays, multilayer implants, visually evoked potentials, brain imaging, neuroprosthetics

\section{Introduction}

Implantable multielectrode arrays (MEAs) have been widely used in neuroprosthetics as means for signal transduction from and to the nervous system [1]-[4]. Microelectrodes can modulate neuronal activity and record spikes or local field potentials in their proximity [5], [6]. As a result of scientific and technological evolution in microengineering and materials science, the field of neuroprosthetics is steadily progressing towards more efficient devices, in particular towards improved interfaces with the biological tissue [7]. A generally desired characteristic of implantable MEAs is a high density of electrodes leading to a high-resolution prosthesis, and in some cases, ideally reaching the single neuron level [8], [9]. High-resolution prostheses can be useful in various applications, such as in artificial vision to replace degenerated photoreceptors with a retinal implant [10][12].

Micro- and nanofabrication technologies offer nowadays the opportunity to manufacture thin and flexible implants with electrode sites in the order of few micrometers [13], [14], meaning that is possible to obtain probes with very high electrode density (up to about 1'000 electrodes $\mathrm{mm}^{-2}$ ). Nevertheless, a limitation associated with the increase of the electrode number and density is the increasing number of traces connecting the array to external electronic devices. In turn, this would augment the implant invasiveness by requiring a wider connecting cable. It is, therefore, necessary to reach a compromise between the size of the probe and number of electrodes.

As an example, most epi-retinal prostheses rely on a trans-scleral cable to connect the implanted electrode array to the extraocular electronic case [15]. A trans-scleral cable is recognized as a weak point because it may cause eye inflammation or leakage through the pars plana incision followed by hypotony [16]. To reduce the risk of such events, the trans-scleral cable has to be limited in its width (in general less than $2 \mathrm{~mm}$ ). In turn, this constraint imposes a limitation on the maximum number of electrodes that can be addressed [17].

In this perspective, multilayer fabrication allows the placement of electrodes with their interconnects on different levels, favoring an increase in electrode density while maintaining a reasonable implant size [18], [19]. An example of multilayer placement is visible in figure 1(a), where three electrodes can be placed on three different layers (bottom) instead of one (top), 
with a slight - and mostly irrelevant - increase in thickness, but an important reduction in width (see red arrows). Nevertheless, a common drawback when using multi-layered probes is the increased and inconsistent distance between the electrodes and the overall surface of the implant (figure 1(b), flat), or in other words, the electrode-tissue distance. This electrode-tissue separation is an important parameter to be minimized for an efficient implantable neuroprosthesis [5], [20]-[22]. In case of neural recordings, the closer the electrogenic cell is to the electrode, the higher is the probability of detecting and specifically identifying its neural activity. In the case of neuronal stimulation, the reduction of the electrode-tissue separation increases the spatial selectivity [23], [24]. Multilayer probes are therefore deprived of a reliable cell-electrode contact, especially for the most bottom levels and when exploiting flexible polymeric materials characterized by relatively thick deposition thicknesses (tens of $\mu \mathrm{m}$ ) due to their high viscosities [25]. Thicker depositions lead to thicker encapsulations and therefore larger electrode-tissue distances.

In order to overcome the above-mentioned issues, we developed a 3D electrode array able to situate the electrodes closer to target cells. With this method, it is possible to manufacture flexible multilayer probes with 3D electrodes flush with the implant surface (figure 1(b), 3D flush) or protruding from it (figure 1(b), 3D protruding). In this article, we describe the details about the fabrication method and show the results with electrochemical characteristics and in vivo recordings of $3 \mathrm{D}$ protruding electrodes.

\section{Methods}

\subsection{MEA Fabrication}

A release layer of TiW $(200 \mathrm{~nm}) / \mathrm{Al}(1 \mu \mathrm{m})$ was deposited using a magnetron sputter onto 4-inch Si wafers. The deposition of a polyimide (PI, PI2611 HD Microsystems) layer of $8 \mu \mathrm{m}$ was obtained by spin-coating at $1750 \mathrm{rpm}$, soft-baking at $65{ }^{\circ} \mathrm{C}$ ( $5 \mathrm{~min})$ and at $95^{\circ} \mathrm{C}(5 \mathrm{~min})$, and hard-baking at $200{ }^{\circ} \mathrm{C}(1 \mathrm{hr})$ and $300{ }^{\circ} \mathrm{C}(1 \mathrm{hr})$ both under nitrogen atmosphere. The patterning of electrodes was performed by magnetron sputtering Ti $(5 \mathrm{~nm}) / \mathrm{Pt}(120 \mathrm{~nm}) / \mathrm{Ti}(5 \mathrm{~nm})$ onto oxygen plasma treated PI, followed by photolithography with a slight photoresist reflow, and ion beam etching. The electrodes were $100 \mu \mathrm{m}$ in diameter with $20 \mu \mathrm{m}$ wide interconnects. After photoresist removal, the substrates were coated again with PI at $6 \mu \mathrm{m}$ (1900 rpm) following the same procedure as described for the first layer. For bilayer implants, the steps of metallization and PI encapsulation were repeated once again, with a PI thickness of $13 \mu \mathrm{m}(1000 \mathrm{rpm})$ for 3D protruding electrodes (otherwise 6 $\mu \mathrm{m})$. At this stage, the fabrication process is as reported in figure 2 (steps $1 \mathrm{a}, 1 \mathrm{~b}$, and $1 \mathrm{c}$ respectively). To produce the openings (either flat or 3D), a $600 \mathrm{~nm}$ Si hard mask was sputtered on the substrates and patterned by photolithography and chlorinebased dry etching using different designs according to the type of electrodes (figure 2, steps $2 \mathrm{a}, 2 \mathrm{~b}$, and $2 \mathrm{c}$ respectively). An opening disc of $92 \mu \mathrm{m}$ in diameter was exposed for flat structures, while an $8 \mu \mathrm{m}$ wide ring with diameters of $76 \mu \mathrm{m}$ (internal) and $92 \mu \mathrm{m}$ (external) for 3D structures. The top PI layer was directionally dry etched using oxygen plasma until the openings reached all of the electrode layers ( $\mathrm{Pt}$ is used as etch stop material, figure 2, steps 3a, 3b, and 3c respectively), then the Si mask was removed by dry etching. Flat electrode arrays do not need further microfabrication steps and can be shaped by laser cutting, released from the wafer by aluminum anodic dissolution, and bonded to a printed circuit board (PCB). The PCB bonding was performed with a silver paste (EPO-TEK H20E-FC, Epoxy Technology) contacting the pads and an insulating epoxy glue (EPO-TEK 302-3M, Epoxy Technology) as encapsulation. Prior the laser cutting and releasing, 3D flush electrodes should undergo one more step of pillar covering by Pt magnetron sputtering $(200 \mathrm{~nm})$ through a $50 \mu \mathrm{m}$ thick rigid stencil with holes aligned on the electrodes (figure 2, step 4d). For 3D protruding electrodes another photolithographic step was necessary to protect electrodes, pillars, and pads (figure 2, step 4c); while directionally oxygen dry etching was performed to reduce the rest of the PI encapsulation layer to a thickness of about $6 \mu \mathrm{m}$ (figure 2, step 5c). The protruding PI pillars of about $13 \mu \mathrm{m}$ high in total (i.e., with 6 to $7 \mu \mathrm{m}$ protrusion) were finally covered with Pt as for flush electrodes, cut, released, and bonded to the PCB for testing and implantation.

\subsection{Microscopy and electrochemical characterization}

The electrode arrays were imaged with light microscopy and scanning electrons microscopy (SEM). Image post-processing was performed using ImageJ.

Electrochemical impedance spectroscopy (IS) was performed with a potentiostat (Compact Stat, Ivium Technologies). The arrays were soaked in phosphate buffered saline (PBS, 1x) together with a Pt counter and an $\mathrm{Ag} / \mathrm{AgCl}$ reference wire, the potential was set at $50 \mathrm{mV}$, and the impedance magnitude and phase were measured between $1 \mathrm{~Hz}$ and $1 \mathrm{MHz}$.

Cyclic voltammetry (CV) was performed with the same setup and three-electrodes configuration as for IS. The applied voltage $\mathrm{V}$ was scanned between -0.6 and $0.8 \mathrm{~V}$ at a rate of $50 \mathrm{mV} \mathrm{s}^{-1}$ and the current was measured and averaged for each electrode (6 cycles, the first cycle was discarded). The cathodic phase area (shaded area in figure 4(c)) was measured to 
determine the charges generated per cycle (area/scan rate) and the normalized cathodic charge storage capacity (CSC, charges/electrode surface area).

\subsection{In vivo experiments}

Experiments were conducted under the animal authorization GE13416 with 2-months old C57BL/6J mice (Charles River). Animals were kept in a $12 \mathrm{~h}$ day/night cycle with access to food and water ad libitum. Experiments were carried out during the day cycle. Before the surgical procedure, mice were anesthetized with isoflurane inhalation (induction $0.8-1.51 \mathrm{~min}^{-1}, 4-5$ $\%$; maintenance $0.8-1.51 \mathrm{~min}^{-1}, 1-2 \%$ ). Analgesia was performed by subcutaneous injection of buprenorphine (Temgesic, $\left.0.1 \mathrm{mg} \mathrm{kg}^{-1}\right)$, and a local subcutaneous injection of lidocaine $\left(6 \mathrm{mg} \mathrm{kg}^{-1}\right)$ and bupivacaine $\left(2.5 \mathrm{mg} \mathrm{kg}^{-1}\right)$ with a 1:1 ratio. The depth of anesthesia was assessed with the pedal reflex, the eyes were protected with artificial tears, and the temperature was maintained at $37^{\circ} \mathrm{C}$ with a heating pad during both surgical and recording sessions.

Deeply anesthetized animals were placed on a stereotaxic frame and the skin was opened. A squared craniotomy of approximately ( 4 x 4) $\mathrm{mm}$ was opened either over the left or right visual cortices (identified by stereotaxic coordinates). The multielectrode array was inserted in the visual cortex using a micromanipulator (SM-15R, Narishige), while a reference stainless-steel screw electrode was placed on the rostral side of the cranium, outside of the visual cortex. A needle placed subcutaneously in the dorsal area near the tail served as the ground. 10 light flashes $\left(4 \mathrm{~ms}, 1-30 \mathrm{~cd} \mathrm{~s} \mathrm{~m}^{-2}\right)$ were delivered with a Ganzfeld stimulator (Biomedica Mangoni) positioned close to the contralateral eye and the corresponding visually evoked cortical potentials (VEPs) were recorded for $1000 \mathrm{~ms}(50 \mathrm{~ms}$ pre-stimulus and $950 \mathrm{~ms}$ post-stimulus) and filtered $(0.1-300$ $\mathrm{Hz}$ ). The signals were acquired simultaneously in four channels connected to four of the electrodes in the probe. VEPs signals were plotted in Matlab R2015b (MathWorks) and peaks amplitudes, prominences, and latencies determined. For the evaluation of the signal-to-noise ratio (SNR), the standard deviation of the baseline was calculated within the first $50 \mathrm{~ms}$ of recordings prior to the flash stimulus (noise, $\mathrm{N}$ ), while the full signal standard deviation was calculated for the entire $1000 \mathrm{~ms}$ of recordings (signal, S).

Animals were euthanized while still under anesthesia by $\mathrm{CO}_{2}$ inhalation. In animals designated for brain imaging, the craniotomy was closed and the animal was left implanted for 1 week before perfusion. Animals were then euthanized with an injection of pentobarbital $\left(150 \mathrm{mg} \mathrm{kg}^{-1}\right)$ under a chemical hood. The chest cavity was open to expose the beating heart and the descending aorta was clamped to deliver the fixative solution only to the brain. A needle was inserted in the left ventricle and the right atrium was cut to allow complete bleeding. The animal was immediately perfused with PBS followed by a fixative solution of $4 \%$ paraformaldehyde (PFA) in PBS. At the end of the procedure, the head of the animal was cut and the brain removed and placed in $4 \%$ PFA for post-fixation.

\subsection{Whole brain imaging}

Brain clarification was performed according to a previously described procedure [26]. Briefly, after overnight post-fixation at $4{ }^{\circ} \mathrm{C}$ in PFA $4 \%$, the brain was immersed in hydrogel solution (Acrylamide $40 \%+$ VA-044 initiator powder in PBS $1 \mathrm{x}$ ) at $4{ }^{\circ} \mathrm{C}$ for three days. The hydrogel polymerization was induced by keeping the sample at $37^{\circ} \mathrm{C}$ for $3 \mathrm{hrs}$, then the brain was passively clarified in $4 \%$ sodium dodecyl sulfate clearing solution $(\mathrm{pH} 8.5)$ for four weeks under gentle agitation at $37^{\circ} \mathrm{C}$.

The whole clarified brain was transferred to Histodenz solution at pH 7.5 (Sigma) and imaged with a light-sheet microscope. To image the electrode array within the mouse brain we used a custom-made light-sheet microscope optimized to image labeled clarified tissue: Clarity Optimized Light-sheet Microscope (COLM), as previously reported (http://tomerlab.org/colm). Briefly, the sample was illuminated by two digitally scanned light-sheets coming from opposite directions. The sample was excited with the $488 \mathrm{~nm}$ laser and emitted fluorescence was collected by high numerical aperture objectives (Olympus XLPLN10XSVMP N.A 0.6) filtered (Brightline HC 525/50, Semrock) and imaged on a digital CMOS camera (Orca-Flash 4.0 LT, Hamamatsu) at a frequency ranging between 5 and $10 \mathrm{fps}$. A self-adaptive positioning of the light sheets across Z-stacks acquisition ensured an optimal image quality over up to $1 \mathrm{~cm}$ of tissue.

\subsection{Statistical analysis}

The computation of the mean, the standard deviation (s.d.) and the standard error of the mean (s.e.m.) of data distributions and the graphical representation of the results were performed with OriginPro 2017G (OriginLab), where $w$ indicates the number of the electrode tested, $n$ is the number of recordings, and $N$ represents the number of animals. Statistical analysis was performed with a t-test or a non-parametric Mann-Whitney test depending on the dataset distribution. 


\section{Results}

The manufacturing method we have proposed to create multilayer 3D electrode arrays applies established wafer-scale processes in the field of microtechnology [27]-[29]. The multilayer 3D electrode arrays can be fabricated in two fashions: with flush or protruding electrodes. Compared to multilayer flat electrodes, the fabrication of 3D flush structures requires a single additional step of pillars covering (step (4d) in figure 2), as the photolithographic and etching steps only differentiate in the masking design. Three additional steps $(4 c, 5 c$, and $6 c$ in figure 2$)$ with respect to multilayer flat electrodes are required to fabricate the 3D protruding pillars of variable heights. The materials used to fabricate the arrays are PI as a substrate and $\mathrm{Pt}$ as a metallic conductor, two widely used materials in implantable medical devices. However, other polymers such as parylene $\mathrm{C}$ and PDMS could be used, as well as other conductive materials and coatings such as gold, iridium oxide, and platinum gray. Figure 1(c) shows a magnified cross-section view of a 3D protruding electrode. From this image, it is clear how the metallization of the pillar wall generates an electrical connection between the based electrode and the top surface of the pillar.

The differences in structure, electrode design, and fabrication process used to manufacture monolayer flat (a), bilayer flat (b), and bilayer 3D flush/protruding (c) electrode arrays is shown in figure 2. It is worth to mention that bilayer arrays have the width (needed for interconnects) almost half the size of monolayer configurations. Consequently, for three layers, the difference would be of about $1 / 3$. The 3D structures have been obtained by etching a ring in the encapsulation layer at the electrode location instead of etching the entire openings (figure 2 steps $2 b$ and $2 c$ ), therefore creating a pillar or cylinder on top of the base electrode. To make the pillar electrically conductive and connecting it with the base electrode, the pillar walls have been coated with Pt by sputtering (figure 2, steps $4 \mathrm{~d}$ and $6 \mathrm{c}$ ), which is known to deposit material relatively isotropically [30]. At this point, the electrode has been elevated to the pillar top surface and at the same level for all the layers. The difference in the fabrication between 3D flush and 3D protruding are two extra steps of photolithography (protecting the pillars, step 4c) and PI dry etching (lowering the overall encapsulation thickness, step 5c). Indeed, for protruding electrodes, one has to deposit a thicker PI encapsulation layer corresponding to the desired pillar height and in prevision of the extra etching step.

A light microscopy image of the bilayer implants in figure 3(a) shows a fabricated eight-electrode array with an electrode diameter of $100 \mu \mathrm{m}$ and a pitch size of $200 \mu \mathrm{m}$. Here, the Pt covering of pillars is perceivable, and the organization of the interconnects is visible through the PI, where they are appropriately aligned and therefore sparing a significant area of the implant. In the following part of the study, we focus only on 3D protruding electrodes. The choice is justified by the fact that their structure, although more complicated in terms of fabrication, may enable a better contact with the tissue [23], [24]. SEM pictures show a tilted view of three protruding electrodes together with a zoom into one of them (figure $3(\mathrm{~b})$ and (c)). The electrode diameter is $100 \mu \mathrm{m}$ with a pillar diameter of $76 \mu \mathrm{m}$, leading to a groove width of $8 \mu \mathrm{m}$. In this electrode array, the pillars have been fabricated with a protruding height of about $6.5 \mu \mathrm{m}$ above the top PI surface (figure 3(d)). It is to notice that, although the layers are placed at a different depth within the PI, the top contact sites for each electrode lay at the same height. In addition, the sputtered Pt covering the pillars (brightest material in figure 3(b) and (c)) surpasses the size of the opening and deposits on the encapsulation layer. This is because the hole design in the stencil had a diameter of $100 \mu \mathrm{m}$, matching the size of the contact at the pillars bottom. The sputtering through the stencil could be replaced with lift-off or other etching processes resulting in more precise pillars covering. Figure 3(e) highlights the surface roughness appeared on the pillar walls after PI dry etching.

Figure 4 illustrates the electrochemical characterization of the fabricated electrode arrays, in a monolayer configuration, consisting of flat and 3D protruding electrodes. The purpose of these measurements is to validate 3D electrodes and establish possible differences with standard flat electrodes. IS (figure 4 (a) and (b)) shows a typical electrode-electrolyte interface behavior [5]. The most prominent difference is the decreased impedance magnitude over the tested range of frequencies for the 3D protruding electrodes $(w=19)$ with respect to the flat electrodes $(w=12)$. In addition, the 3D protruding electrodes show a more resistive behavior at higher frequencies $\left(10^{4}-10^{6} \mathrm{~Hz}\right)$. The reason for lower impedance values can be attributed to the difference in the electrode surface area: $0.0111 \mathrm{~mm}^{2}$ for $3 \mathrm{D}$ protruding and $0.0084 \mathrm{~mm}^{2}$ for flat electrodes. At $1 \mathrm{kHz}$, the impedance magnitude values (mean \pm s.d.) are $194 \pm 47 \mathrm{kOhm}$ for 3D protruding electrodes and $427 \pm 155 \mathrm{kOhm}$ for flat electrodes $\left(p<0.0001\right.$, t-test), while the values for the phase are not significantly different at this frequency $\left(-65.7 \pm 4.0^{\circ}\right.$ for 3D protruding electrodes and $-63.2 \pm 7.7^{\circ}$ for flat electrodes; $p=0.244$, t-test). Electrochemical IS also showed no major differences between the bottom and top layers for 3D protruding electrodes in bilayer probes ( $p=0.45$, Mann-Whitney test).

$\mathrm{CV}$ has been performed (figures $4(\mathrm{c})$ and $(\mathrm{d}))$ within the Pt water window $(-0.6$ to $0.8 \mathrm{~V})$ for $3 \mathrm{D}$ protruding electrodes $(w=$ 18 ) with respect to flat electrodes $(w=12)$. It is visible in figure $4(\mathrm{c})$ that the measured currents extend to greater values for the 3D geometry. As for the impedance, this effect derives from an increased surface area, enabling a less impeded charge injection at the electrode-electrolyte interface [5]. Accordingly, the charges injected per cycle and their corresponding CSC are calculated for the cathodic phase from the area within the curves (figure 4(d)). The amount of charges generated for each cycle is greatly increased for the 3D protruding electrodes $(0.301 \pm 0.024 \mu \mathrm{C}$ for $3 \mathrm{D}$ and $0.197 \pm 0.022 \mu \mathrm{C}$ for flat; $p<0.0001$, Mann-Whitney test). Interestingly, when normalized by the exposed surface area, the CSC of $3 \mathrm{D}$ structures remains slightly higher $(2.71 \pm 0.22$ $\mathrm{mC} \mathrm{cm}{ }^{-2}$ for 3D and $2.34 \pm 0.26 \mathrm{mC} \mathrm{cm}^{-2}$ for flat; $p<0.01$, Mann-Whitney test), even though it should not differ, as it is made 
of the same material. This difference could be due to the roughness of the pillar walls appearing after PI dry etching, which further increases the surface area of 3D electrodes. Figure 3(e) provides an SEM picture of the Pt-coated roughness on a pillar wall. Table 1 displays the values mentioned above of surface areas, cathodic charges (from CV), and cathodic CSC for both 3D protruding and flat electrodes. Moreover, it shows the absolute and percent differences of the measurements together with the percent quantity that is not due to the geometrical surface area of the electrodes (32\%), but it likely represents the roughness contribution. As the calculated increase from the charges $(21 \%)$ slightly differs from the amount obtained directly from the cathodic CSC difference (16\%), we inserted in the table the average value between these two, which represents a probable increase in active surface area due to roughness (18.5\%). Accounting this, the more realistic increment in the active surface area from flat to $3 \mathrm{D}$ electrodes is about $50 \%$, which further augments the electrode-electrolyte contact area, contributing to the increase in charge storage and to the decrease of impedance.

Table 1. Values for surface area, charges generated during the cathodic phase of CV, and cathodic CSC for the 3D protruding and flat electrodes. The absolute and percent differences between the two types of electrodes are also shown. The last column shows the difference percent that cannot be attributed to the $3 \mathrm{D}$ geometry, therefore likely due to roughness.

\begin{tabular}{cccccc}
\cline { 2 - 5 } & $3 \mathrm{D}$ & Flat & Difference & $\begin{array}{c}\text { Difference } \\
{[\%]}\end{array}$ & $\begin{array}{c}\text { Roughness } \\
\text { contribution } \\
{[\%]}\end{array}$ \\
\hline $\begin{array}{c}\text { Surface area } \\
{\left[\mathrm{mm}^{2}\right]}\end{array}$ & 0.0111 & 0.0084 & 0.0027 & 32 & \\
\hline $\begin{array}{c}\text { Charges } \\
{[\mu \mathrm{C}]}\end{array}$ & 0.301 & 0.197 & 0.104 & $\sim 53$ & $53-32=21$ \\
\hline $\begin{array}{c}\mathrm{CSC} \\
{\left[\mathrm{mC} \mathrm{cm}^{-2}\right]}\end{array}$ & 2.710 & 2.337 & 0.373 & $\sim 16$ & 16 \\
\hline Average $[\%]$ & & & & & 18.5 \\
\hline
\end{tabular}

Bi-layered 3D protruding probes have been used to record VEPs in mice using a ganzfeld flash stimulation. Figure 5(a) shows a sketch of the experiment with the implant location and approximate depth into the visual cortex of the eight-electrode array. The insertion of the probe was stopped once the last electrode disappeared into the brain surface, therefore - assuming that the implant did not bend inside the brain - we can approximate the location of each electrode using the array pitch size of $200 \mu \mathrm{m}$. Hence, the different recording depths were at about 100, 300, 500, 700, 900, 1100, 1300, and $1500 \mu \mathrm{m}$ below the cortical surface. The mouse visual cortex is about $1.2-1.3 \mathrm{~mm}$ thick [31], [32], meaning that the two terminal electrodes (at $1300 \mu \mathrm{m}$ and $1500 \mu \mathrm{m})$ could presumably lay in the white matter or the transition region. Nevertheless, considering that a perfectly vertical and straight insertion of a flexible implant hardly represents the real scenario, most likely at least one of these electrodes is still found in the deep layers of the visual cortex. Figure 5(b) shows an example of VEP recordings of all the eight electrodes upon a flash illumination of $30 \mathrm{~cd} \mathrm{~s} \mathrm{~m}^{-2}$. Each trace represents the average of $n=10$ consecutive recordings $(1 \mathrm{~Hz}$ repetition rate). Peaks characteristic of the VEPs (N1, N2, and N3 respectively) are visible at 50 - 90 ms, as well as $100 \mathrm{~ms}$, and $110-150 \mathrm{~ms}$ post-stimulus [33]. Peak amplitude and latency shift as the recordings were taken more profound in the cortex. The amplitude (in case of N1, N3) and polarity (in case of N2) of the electrode at $1500 \mu \mathrm{m}$ are very dissimilar from the others, probably because it is located below the cortical layers. For better visualization and comparison of depth effects, the traces of the first four electrodes $(100-700 \mu \mathrm{m})$ are enlarged and superimposed in figure 5(c). Negative (N1, N2, N3) and positive peaks (P1, P2) are identified. As the depth into the cortex increases (black arrows): N1 decreases its amplitude, while $\mathrm{N} 2$ increases it. In panel (d), the mean ( \pm s.e.m.) amplitude values of N1 and N2 are plotted versus the penetration depth $(N=$ 3).

VEP traces of the eight electrodes recorded at light intensities of 1, 5, 10, and $30 \mathrm{~cd} \mathrm{~s} \mathrm{~m}^{-2}$ are presented in figure 6(a-d), and $\mathrm{N} 1$ and N2 latencies and prominences have been identified and plotted versus the light intensity in figure 6(e-h). The characteristic evolution of the VEP peaks can be observed. The increase of N1 amplitude and the decrease of latency with light intensity, as well as the opposite behavior of N1 and N2 amplitudes with recording depth are known phenomena of local field potentials recorded in the visual cortex [33]-[35]. Upper and lower regions of the cortex approximately follow the same trend, apart from N1 prominences (panel $(\mathrm{g})$ ), where the upper region $(100-700 \mu \mathrm{m})$ responses continuously augment with light intensity, which is a contrasting behavior concerning the other prominence trends.

Analyzing single sweep recordings, it is possible to observe clearer and more defined peaks with $3 \mathrm{D}$ structures with respect to flat electrodes, where the peaks are less evident and not unequivocally identifiable (figure 7(a)). To further characterize the SNR of the 3D electrodes compared to the flat ones, the s.d. of the single recording traces of each type of electrodes have been calculated for the full signal (S) and the pre-stimulus baseline (N) (figure 7(b)). The signal after the light stimulus has larger 
peaks for 3D protruding electrodes with respect to flat $(174 \pm 39.7 \mu \mathrm{V}$ for $3 \mathrm{D}$ protruding electrodes and $133 \pm 23.7 \mu \mathrm{V}$ for flat electrodes; $p<0.0001$, Mann-Whitney test), while the baseline has a lower s.d., indicating a lower noise level (56 $\pm 32.9 \mu \mathrm{V}$ for 3D protruding electrodes and $75 \pm 19.3 \mu \mathrm{V}$ for flat electrodes; $p<0.0001$, Mann-Whitney test). The s.d. of the full signals divided by the one of baselines (SNR) gives 3.11 for the 3D protruding electrodes and 1.77 for the flat electrodes.

During surgical implantation, the 3D protruding electrodes may be damaged by frictions between the protruding pillar and the brain parenchyma. To verify the integrity of the 3D protruding electrodes within the tissue, the arrays have been imaged inside a clarified mouse brain using a light-sheet microscope. Figure 7(c) contains a photograph of the transparent brain with two implanted 3D electrode arrays used to image the structures as shown in panel (d). In this micrograph, the 3D protruding electrode is recognizable, intact, within the brain tissue (black in the image). For comparison, figure 7(e) illustrates a similar electrode imaged with a light microscope prior to implantation.

\section{Discussion}

In this article, we have proposed an electrode array design that can be used to optimize neural implant with high electrode density, reduced probe size, and small electrode-tissue distance. Multilayer 3D electrode arrays do not require a further significant amount of resources and fabrication steps with respect to flat electrodes; however, their contribution to recording and stimulation capabilities could be significant. The 3D protruding pillars could better reach target neurons separated from the stimulating or recording sites by the fibrotic tissue surrounding the implant [24], [36]. An additional advantageous feature of these cylindrical electrodes (both flush and protruding) is that all sites have the same elevation concerning the global thickness of the implant, independently of the different planes at which they were placed originally with their respecting traces. Moreover, there is no need for electroplated electrodes that are difficult to control, demand considerable material deposition, and rigidify the implant [37].

The proposed fabrication method allows the production of pillar protrusion heights between 0 (flush) and $30 \mu \mathrm{m}$ for up to four electrode layers. Two major aspects have to be considered when fabricating geometries with challenging aspect ratios, as in the case of pillar height to groove width ratios above 3. First, the polyimide at the bottom of the groove could be challenging to etch away even with high over-etching times; secondly, the sputtered covering could not be as homogeneous as for lower aspect ratio geometries, and this could lead to poor electrical contact between the bottom and the top of the pillars. Another factor that limits the number of layers is that the upper levels finish the polyimide etching to create the grooves before the underlying layers. This means that the Pt, acting as an etch stop layer, has to withstand the plasma treatment until all of the bottom levels are entirely etched. Although Pt is a suitable etch stop material for oxygen plasma [38], the directional ion bombardment could damage or thin the Pt after a while. Possible ways to circumvent this problem are either to augment the thickness of Pt depositions to compensate the loss during etching or to deposit another etch stop layer after $\mathrm{Pt}$ (such as $\mathrm{Si}$ or $\mathrm{SiO}_{2}$ ) and remove it before the covering of the pillars.

Here, PI and Pt were used as insulating and conducting material respectively, but other insulators and conductors can be also used to fabricate these electrode arrays.

In general, low impedance electrodes are preferred for recording neural activity, while electrodes with high charge storage/injection capacity are advantageous for stimulation [5]. As they both are influenced by the electrode-electrolyte contact, increasing the surface area (i.e., porosity, roughness, geometry) improves these two characteristics. As visible from electrochemical measures, 3D electrodes provide a better option compared to flat ones for both recording and stimulation.

In vivo recordings of VEPs with 3D protruding electrodes showed a better SNR with respect to flat electrodes, thus allowing a detailed characterization of all the VEP peaks depending on the brain depth and the light intensities.

High-resolution imaging of multielectrode arrays within the brain showed that the $3 \mathrm{D}$ protruding electrodes were perfectly intact after one week of implantation.

In conclusion, 3D electrode arrays allow a multilayer placement of their interconnects within a flexible substrate, thus reducing the size of the probe per the same number of electrodes. In addition, all the electrode sites are maintained at the same level and nearer to the tissue. The surface area of the electrodes is increased by about $50 \%$ compared to flat standard electrodes, which leads to lower electrochemical impedance and higher charge storage capacity. 


\section{Acknowledgments}

This work has been supported by École Polytechnique Fédérale de Lausanne and Medtronic. The authors would like to thank the staff of the EPFL Center of Micronanotechnology (CMi) and Bastien Duckert for their support related to microfabrication processes. We would like to thank also the Imaging and Light Microscopy Facility of the Wyss Center for Bio- and Neuroengineering and Elodie Geneviève Meyer for their help with light-sheet microscopy.

\section{Authors contribution}

M.J.I.A.L. designed the fabrication strategy, fabricated the samples, performed the characterizations, and analyzed the data. P.V. performed the in vivo validation and the whole-brain imaging. D.G. supervised the study. M.J.I.A.L. wrote the manuscript. P.V. and D.G. edited the manuscript.

\section{References}

[1] Fekete $\mathrm{Z}$ and Pongrácz A Multifunctional soft implants to monitor and control neural activity in the central and peripheral nervous system: A review 2017 Sensors Actuators, B Chem. 243 1214-1223.

[2] Wurth S, Capogrosso M, Raspopovic S, Gandar J, Federici G, Kinany N, Cutrone A, Piersigilli A, Pavlova N, Guiet R, et al. Longterm usability and bio-integration of polyimide-based intra-neural stimulating electrodes 2017 Biomaterials 122 114-129.

[3] Weiland JD, Liu W, and Humayun MS Retinal Prosthesis 2005 Annu. Rev. Biomed. Eng. 71 361-401.

[4] Guex AA, Vachicouras N, Hight AE, Brown MC, Lee DJ, and Lacour SP Conducting polymer electrodes for auditory brainstem implants 2015 J. Mater. Chem. B 325 5021-5027.

[5] Cogan SF Neural Stimulation and Recording Electrodes 2008 Annu. Rev. Biomed. Eng. $101275-309$.

[6] Cheung KC Implantable microscale neural interfaces 2007 Biomed. Microdevices $96923-938$.

[7] Lee JH, Kim H, Kim JH, Lee S-H, Hoon Lee J, Kim H, Hun Kim J, and Lee S-H Soft implantable microelectrodes for future medicine: prosthetics, neural signal recording and neuromodulation 2016 Lab Chip 166 959-976.

[8] Nordhausen CT, Maynard EM, and Normann RA Single unit recording capabilities of a 100 microelectrode array 1996 Brain Res. 726 1-2 129-140.

[9] Buzsáki G, Anastassiou CA, and Koch C The origin of extracellular fields and currents--EEG, ECoG, LFP and spikes. 2012 Nat. Rev. Neurosci. 13 6 407-20.

[10] Rodger DC, Fong AJ, Li W, Ameri H, Ahuja AK, Gutierrez C, Lavrov I, Zhong H, Menon PR, Meng E, et al. Flexible parylenebased multielectrode array technology for high-density neural stimulation and recording 2008 Sensors Actuators B Chem. 1322449 460.

[11] Goetz GA and Palanker D V Electronic approaches to restoration of sight 2016 Reports Prog. Phys. 79996701.

[12] Waschkowski F, Hesse S, Rieck AC, Lohmann T, Brockmann C, Laube T, Bornfeld N, Thumann G, Walter P, Mokwa W, et al. Development of very large electrode arrays for epiretinal stimulation (VLARS) 2014 Biomed. Eng. Online 13 11-15.

[13] Multiplexed, High Density Electrophysiology with Nanofabricated Neural Probes 2011 PLoS One 610 e26204.

[14] Tsai D, Sawyer D, Bradd A, Yuste R, and Shepard KL A very large-scale microelectrode array for cellular-resolution electrophysiology 2017 Nat. Commun. 81.

[15] Luo YH-L and da Cruz L The Argus $®$ II Retinal Prosthesis System 2016 Prog. Retin. Eye Res. 50 89-107.

[16] Özmert E and Demirel S Endoscope-Assisted and Controlled Argus II Epiretinal Prosthesis Implantation in Late-Stage Retinitis Pigmentosa: A Report of 2 Cases 2016 Case Rep. Ophthalmol. 73 315-324.

[17] Zrenner E Artificial vision: Solar cells for the blind 2012 Nat. Photonics $66344-345$.

[18] Tooker A, Tolosa V, Shah KG, Sheth H, Felix S, Delima T, and Pannu S Optimization of multi-layer metal neural probe design, in Proceedings of the Annual International Conference of the IEEE Engineering in Medicine and Biology Society, EMBS, 2012, 59955998.

[19] Suaning GJ, Schuettler M, Ordonez JS, and Lovell NH Fabrication of multi-layer, high-density micro-electrode arrays for neural stimulation and bio-signal recording, in Proceedings of the 3rd International IEEE EMBS Conference on Neural Engineering, 2007, 5-8.

[20] Joye N, Schmid A, and Leblebici Y Electrical modeling of the cell-electrode interface for recording neural activity from high-density microelectrode arrays 2009 Neurocomputing 73 1-3 250-259.

[21] Wang J, Thow XY, Wang H, Lee S, Voges K, Thakor N V., Yen SC, and Lee C A Highly Selective 3D Spiked Ultraflexible Neural (SUN) Interface for Decoding Peripheral Nerve Sensory Information 2017 Adv. Healthc. Mater. 1700987.

[22] Spira ME and Hai A Multi-electrode array technologies for neuroscience and cardiology 2013 Nat. Nanotechnol. $8283-94$.

[23] Palanker D, Vankov A, Huie P, and Baccus S Design of a high-resolution optoelectronic retinal prosthesis 2005 J. Neural Eng. 21 S105.

[24] Flores T, Lei X, Huang T, Lorach H, Dalal R, Galambos L, Kamins T, Mathieson K, and Palanker D Optimization of pillar electrodes in subretinal prosthesis for enhanced proximity to target neurons 2018 J. Neural Eng. 153036011.

[25] Lawrence CJ The mechanics of spin coating of polymer films 1988 Phys. Fluids 31 10 2786-2795.

[26] Lee E, Choi J, Jo Y, Kim JY, Jang YJ, Lee HM, Kim SY, Lee HJ, Cho K, Jung N, et al. ACT-PRESTO: Rapid and consistent tissue clearing and labeling method for 3-dimensional (3D) imaging 2016 Sci. Rep. 6 1-13.

[27] Cheung KC Implantable microscale neural interfaces 2007 Biomed. Microdevices 96 923-938.

[28] Rodger DC, Li W, Fong AJ, Ameri H, Meng E, Burdick JW, Roy RR, Edgerton VR, Weiland JD, Humayun MS, et al. Flexible microfabricated parylene multielectrode arrays for retinal stimulation and spinal cord field modulation, in Proc. 4th International IEEE-EMBS Special Topic Conference on Microtechnologies in Medicine and Biology, 2006, 31-34. 
[29] Boppart SA, Wheeler BC, and Wallace CS A Flexible Perforated Microelectrode Array for Extended Neural Recordings 1992 IEEE Trans. Biomed. Eng. $39137-42$.

[30] Kilchenmann SC, Rollo E, Bianchi E, and Guiducci C Metal-coated silicon micropillars for freestanding 3D-electrode arrays in microchannels 2013 Sensors Actuators B Chem. 185 713-719.

[31] Manuel M, Georgala PA, Carr CB, Chanas S, Kleinjan DA, Martynoga B, Mason JO, Molinek M, Pinson J, Pratt T, et al. Controlled overexpression of Pax6 in vivo negatively autoregulates the Pax6 locus, causing cell-autonomous defects of late cortical progenitor proliferation with little effect on cortical arealization. 2007 Development 134 345-55.

[32] Ji W, Gămănuţ R, Bista P, D'Souza RD, Wang Q, and Burkhalter A Modularity in the Organization of Mouse Primary Visual Cortex. 2015 Neuron $873632-43$.

[33] Ridder WH and Nusinowitz S The visual evoked potential in the mouse-Origins and response characteristics 2006 Vision Res. $466-$ 7 902-913.

[34] Heynen AJ and Bear MF Long-term potentiation of thalamocortical transmission in the adult visual cortex in vivo. $2001 \mathrm{~J}$. Neurosci. 2124 9801-13.

[35] Porciatti V, Pizzorusso T, and Maffei L The visual physiology of the wild type mouse determined with pattern VEPs 1999 Vision Res. 3918 3071-3081.

[36] Polikov VS, Tresco PA, and Reichert WM Response of brain tissue to chronically implanted neural electrodes 2005 J. Neurosci. Methods 148 1 $1-18$.

[37] Ordonez JS, Boehler C, Schuettler M, and Stieglitz T Improved polyimide thin-film electrodes for neural implants 2012 Proc. Annu. Int. Conf. IEEE Eng. Med. Biol. Soc. EMBS 5134-5137.

[38] Williams KR, Gupta K, and Wasilik M Etch rates for micromachining processing - Part II 2003 J. Microelectromechanical Syst. 126 761-778. 
a

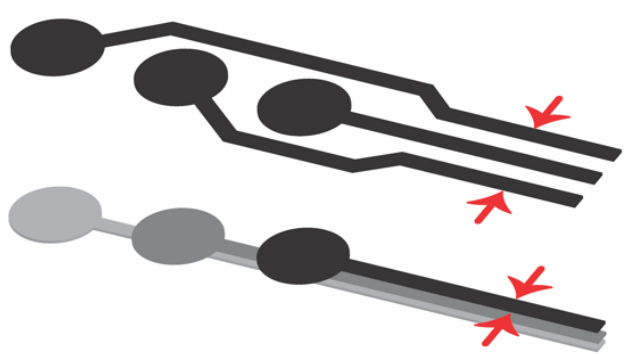

c
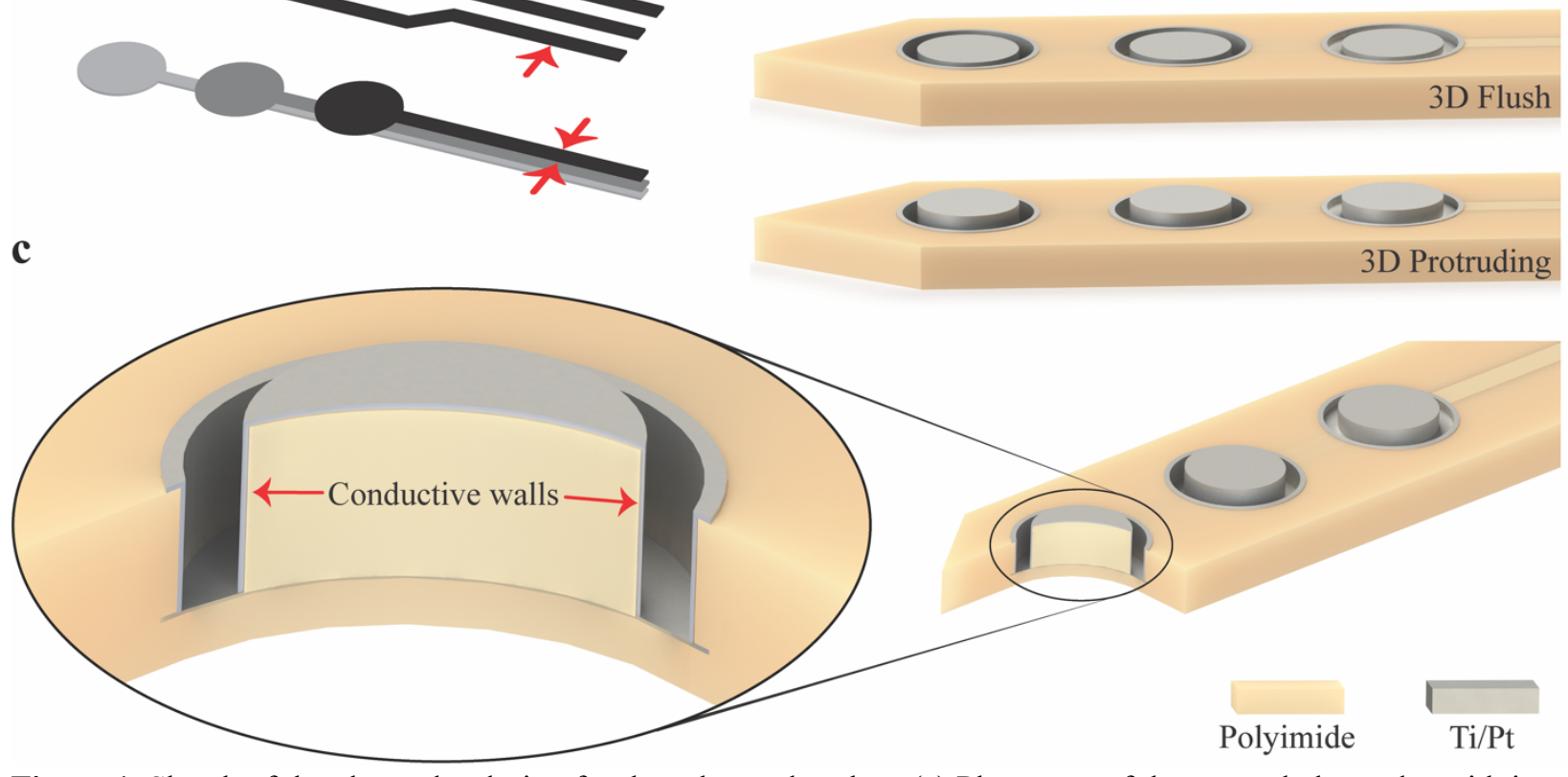

Figure 1. Sketch of the electrodes design for three-layered probes. (a) Placement of three metal electrodes with interconnects in the case of a monolayer (top) and a three-layer (bottom) configuration. Red arrows indicate the width dedicated to interconnects. (b) Drawing of standard flat (top), 3D flush (middle), and 3D protruding (bottom) three-layered electrode arrays. (c) Crosssection and magnification of a 3D protruding electrode. 
$\mathbf{a}$

Monolayer Flat

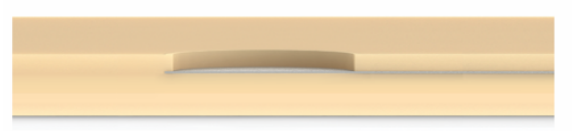

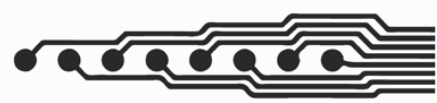

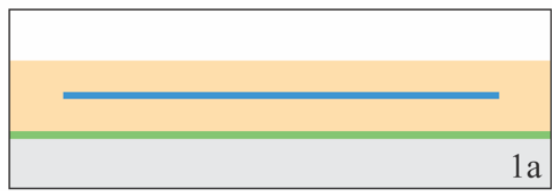

$1 \mathrm{a}$
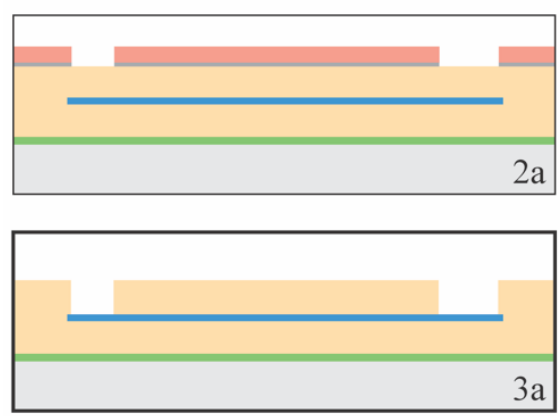

$3 \mathrm{a}$

Si wafer

TiW/Al

Polyimide

$\mathrm{Ti} / \mathrm{Pt}$

Photoresist

Si mask b
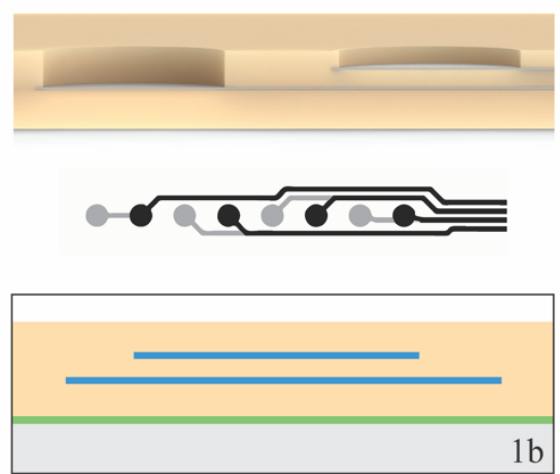

$1 b$
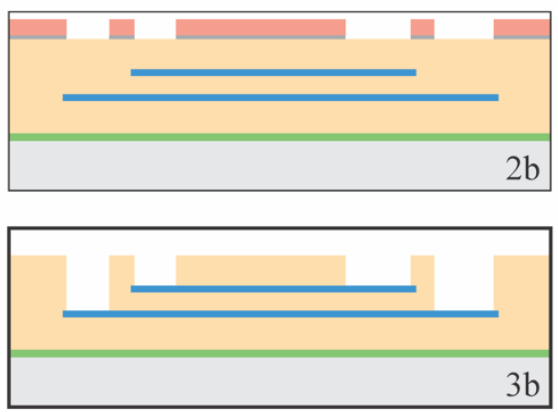

$3 \mathrm{~b}$

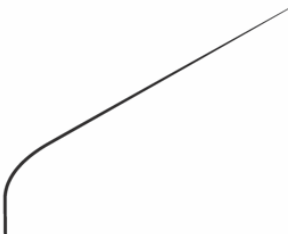

Directly from step

(3c) to step (4d) for

3D flush electrodes.

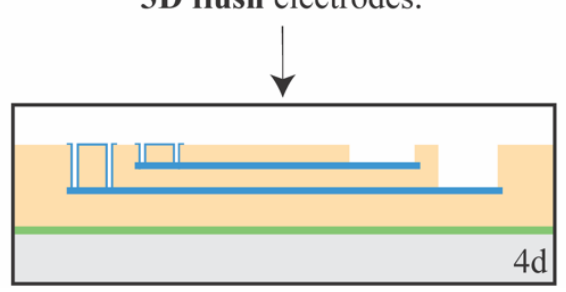

c Bilayer $3 \mathrm{D}$ protruding
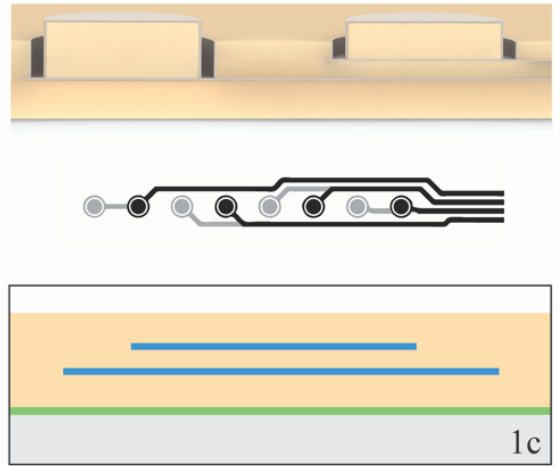

$1 \mathrm{c}$
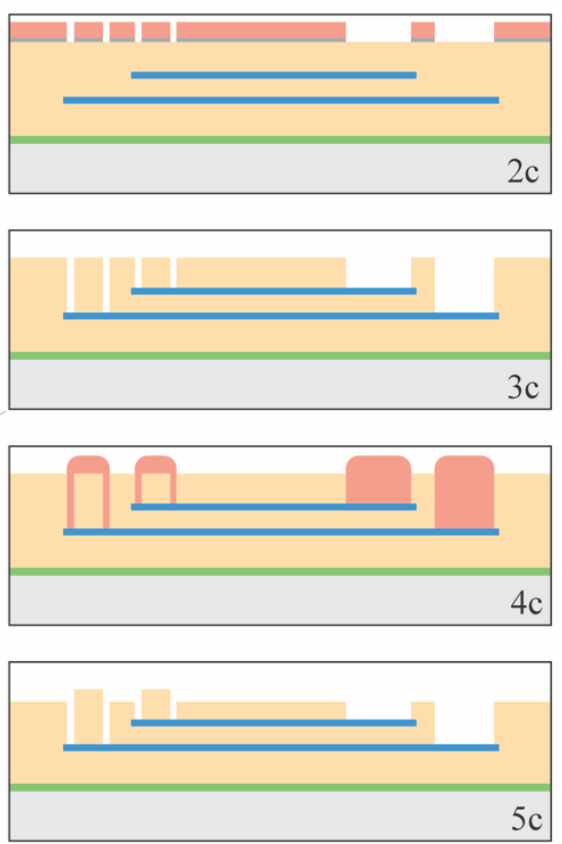

$5 \mathrm{c}$

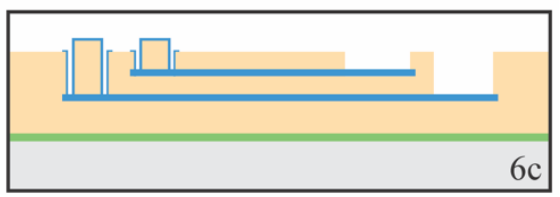

Figure 2. Fabrication process flow for monolayer flat (a), bilayer flat (b), and bilayer 3D electrodes (c). Each column contains a schematic cross-section of the final electrodes (top), a top view of the used Pt design with the bottom layer marked in grey (second row), and the last main steps of the fabrication for the respective structures (following rows). (1a-c): deposition of all PI and Pt layers and patterning of all the Pt layers; (2a-c): hard mask deposition, photolithography, and hard mask patterning; (3a-c): PI dry etching and mask removal; (4c): second photolithography; (5c): second PI dry etching; (6c) and (4d): pillars covering by Pt sputtering through a stencil. 

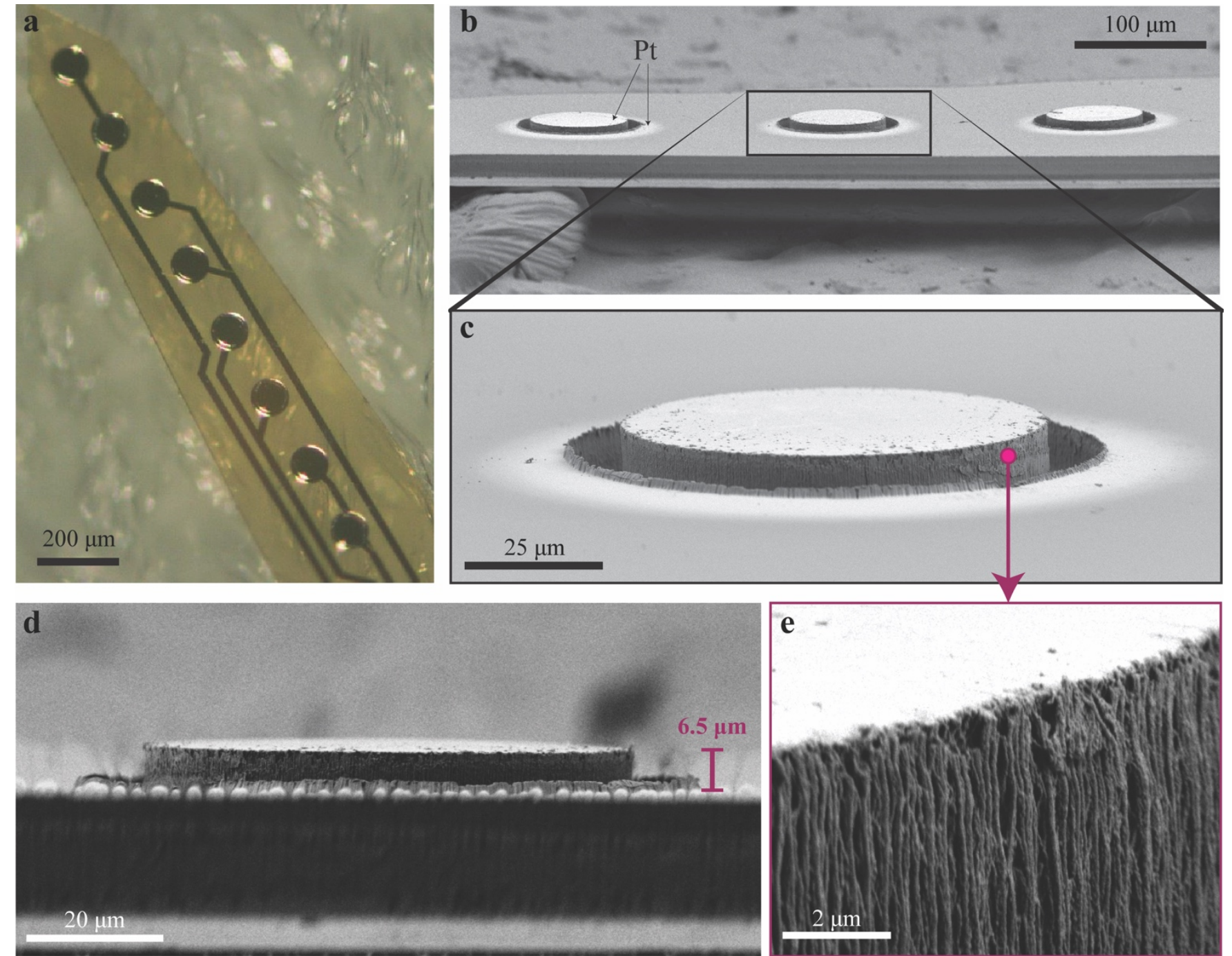

Figure 3. Micrographs of the fabricated bilayer probes. (a) Light microscopy image showing the 3D protruding electrodes and their Pt interconnects placed on two different layers and vertically aligned within PI. (b) SEM image of the 3D protruding electrodes (light colored material: Pt. Tilting angle of $14^{\circ}$ with respect to the side view). (c) Magnification over one 3D protruding electrode. (d) The same electrode looked by its side view, where the protruding height of $6.5 \mu \mathrm{m}$ is visible. (e) Further zoom on the Pt-coated pillar revealing the side wall roughness. 


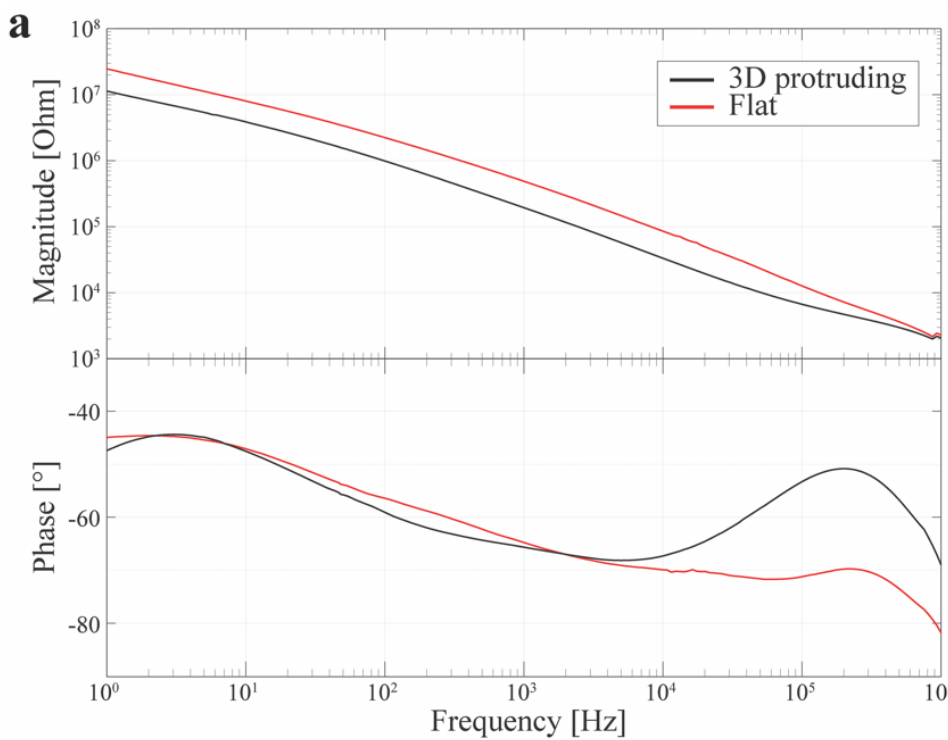

b
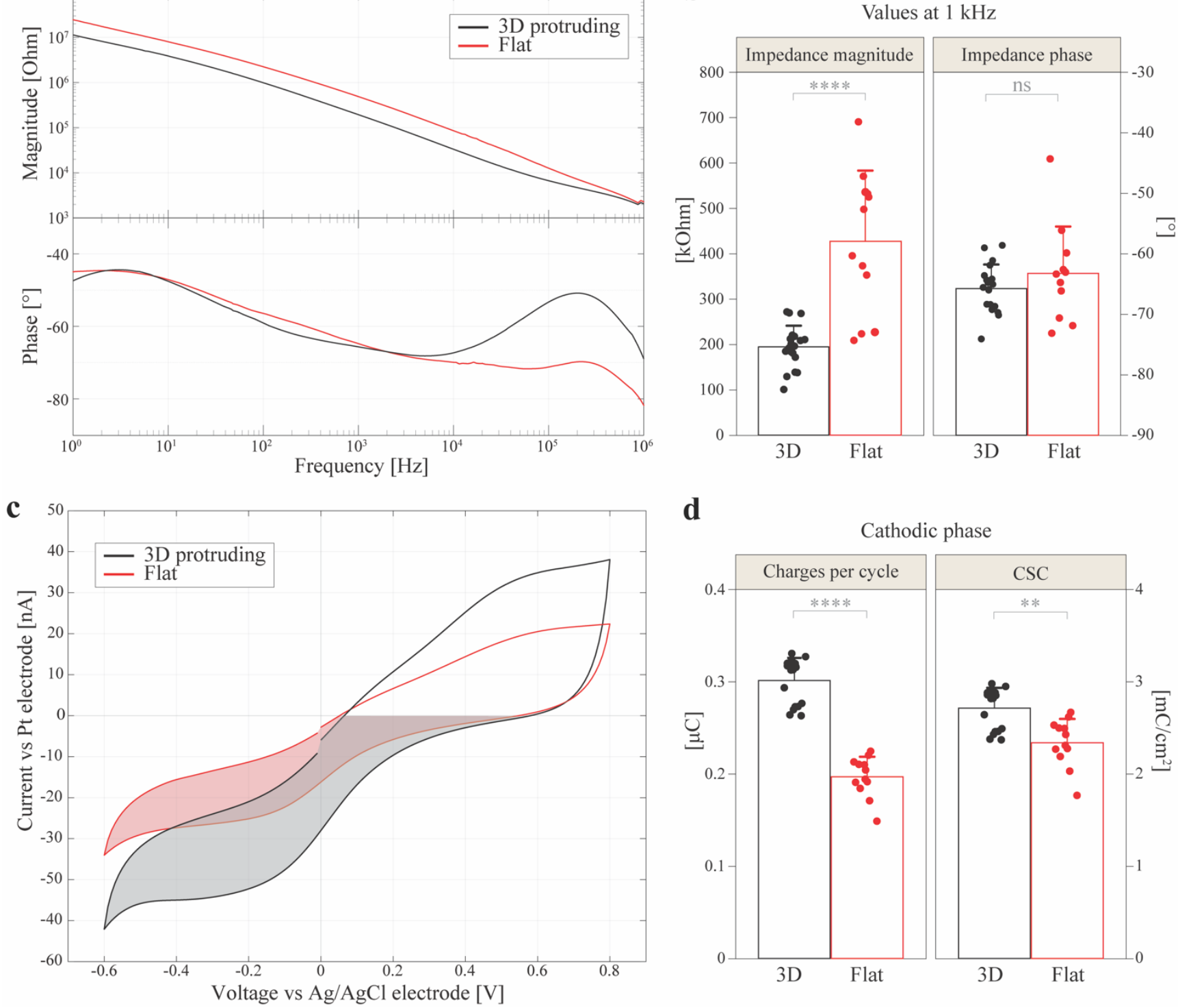

d

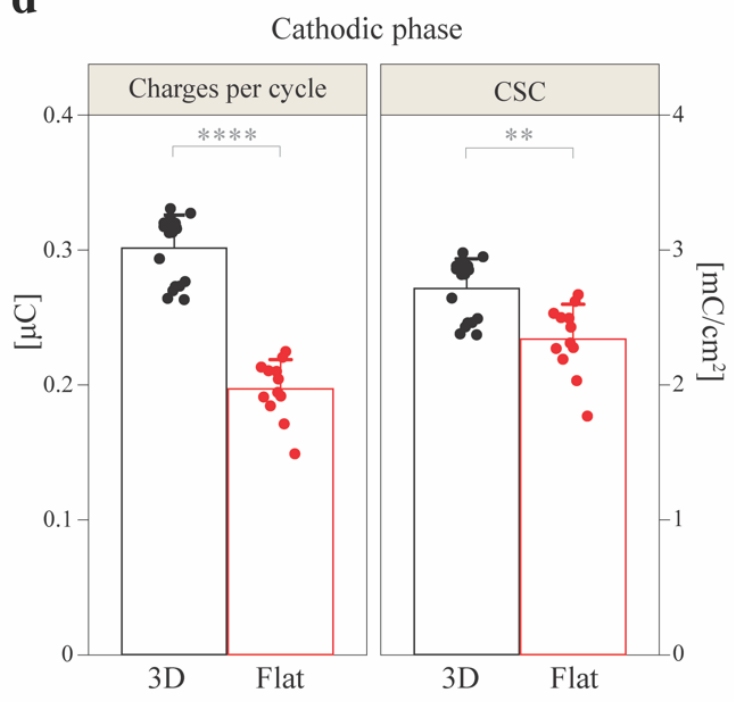

Figure 4. Electrochemical characterizations of 3D protruding and flat electrodes in PBS. (a) Bode plots of IS with the average magnitude (top) and average phase (bottom) for 3D protruding electrodes $(\mathrm{w}=19)$ in black and flat electrodes $(\mathrm{w}=12)$ in red. (b) Quantification (mean \pm s.d.) of the impedance magnitude (t-test, $\mathrm{p}<0.0001$ ) and phase (t-test, $\mathrm{p}=0.244$ ) at $1 \mathrm{kHz}$. (c) Mean CV curves for 3D protruding electrodes (black, $w=18$ ) and flat ones (red, $w=12$ ), measured with a scan rate of $50 \mathrm{mV}$ $\mathrm{s}^{-1}$. (d) Quantification (mean \pm s.d.) of the charges per cycle (Mann-Whitney test, $\mathrm{p}<0.0001$ ) and CSC for the cathodic phase (Mann-Whitney test, $\mathrm{p}<0.01$ ) represented by the shaded area in $(\mathrm{c})$. 
a
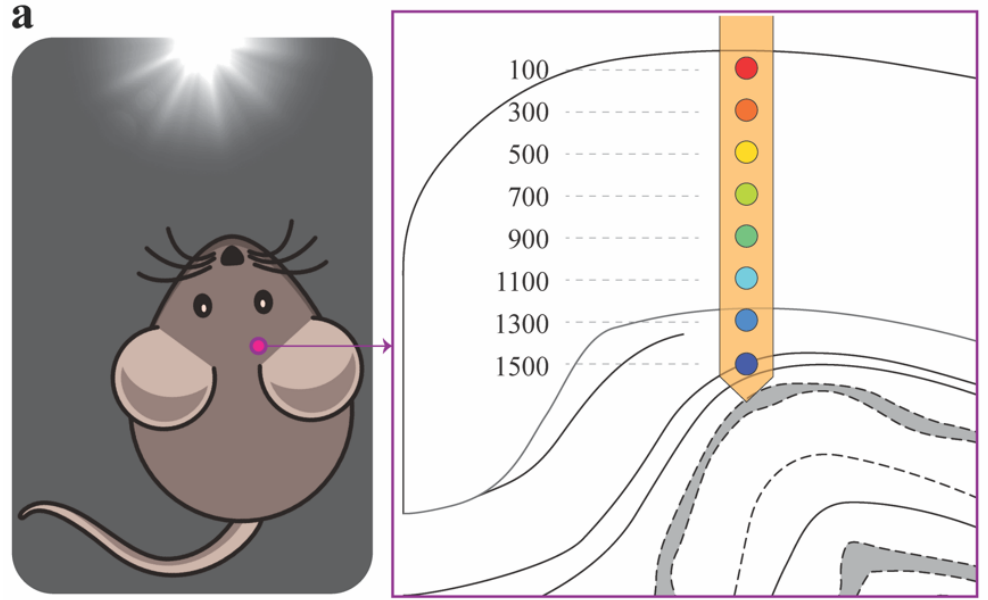

b

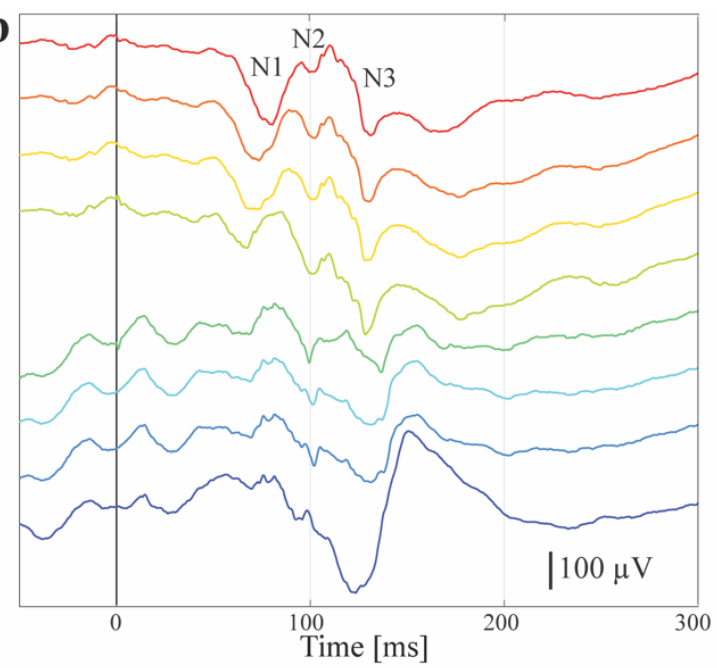

c

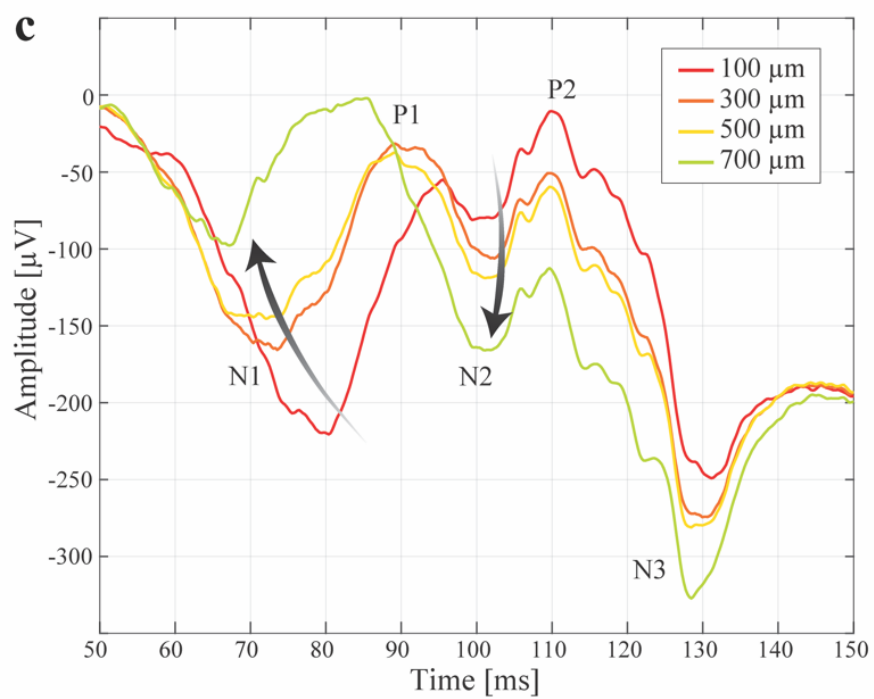

d

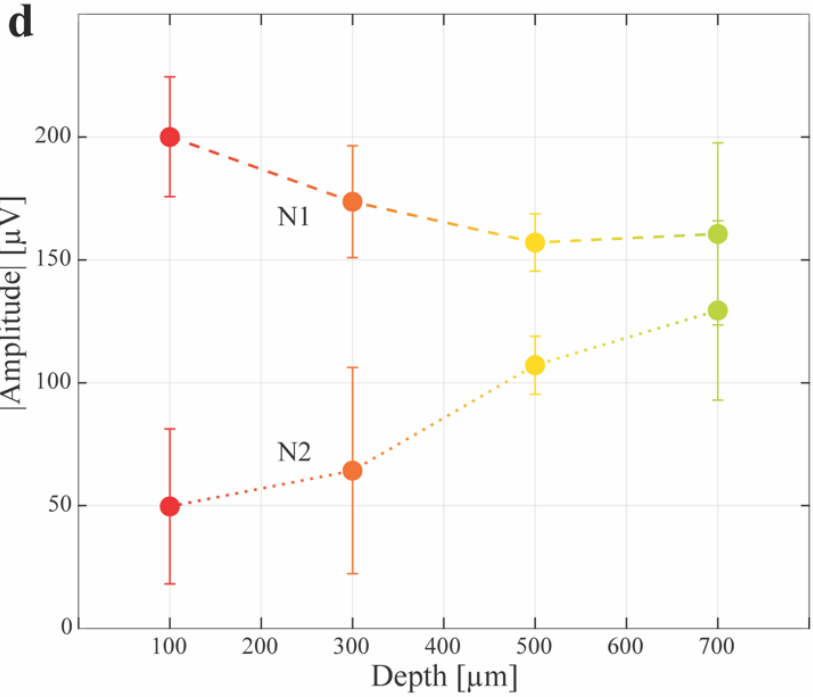

Figure 5. In vivo recordings with $3 \mathrm{D}$ protruding electrodes of VEPs. (a) Sketch of the experimental setting and probe implantation with the approximate depth into the visual cortex in $\mu \mathrm{m}$. (b) Example of VEP recordings with a light intensity of $30 \mathrm{~cd} \mathrm{~s} \mathrm{~m}^{-2}$ (each trace is an average of $\mathrm{n}=10$ repetitions). The vertical black line is the occurrence of the flash. (c) Expansion and superimposition of the traces of the first four electrodes of the graph in (b) $(100-700 \mu \mathrm{m})$. Here, the arrows highlight the evolution of $\mathrm{N} 1$ and $\mathrm{N} 2$ peaks. (d) Absolute amplitude values of $\mathrm{N} 1$ and $\mathrm{N} 2$ peaks plotted versus the penetration depth $(\mathrm{N}=3$, mean \pm s.e.m.; for each animal $n=10$ repetitions have been averaged). 

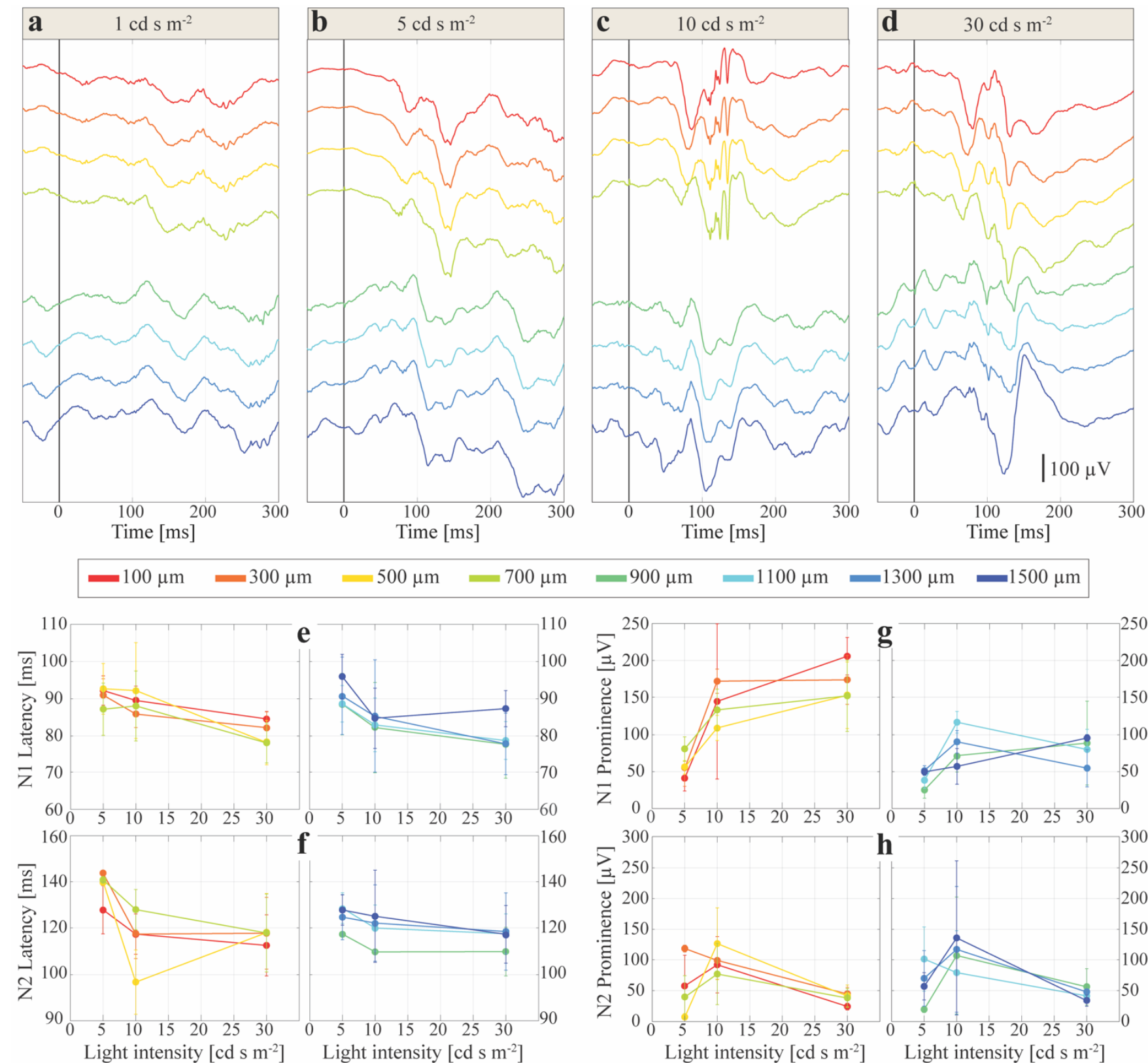

$-900 \mu \mathrm{m} \quad-1100 \mu \mathrm{m} \quad-1300 \mu \mathrm{m} \quad-1500 \mu \mathrm{m}$
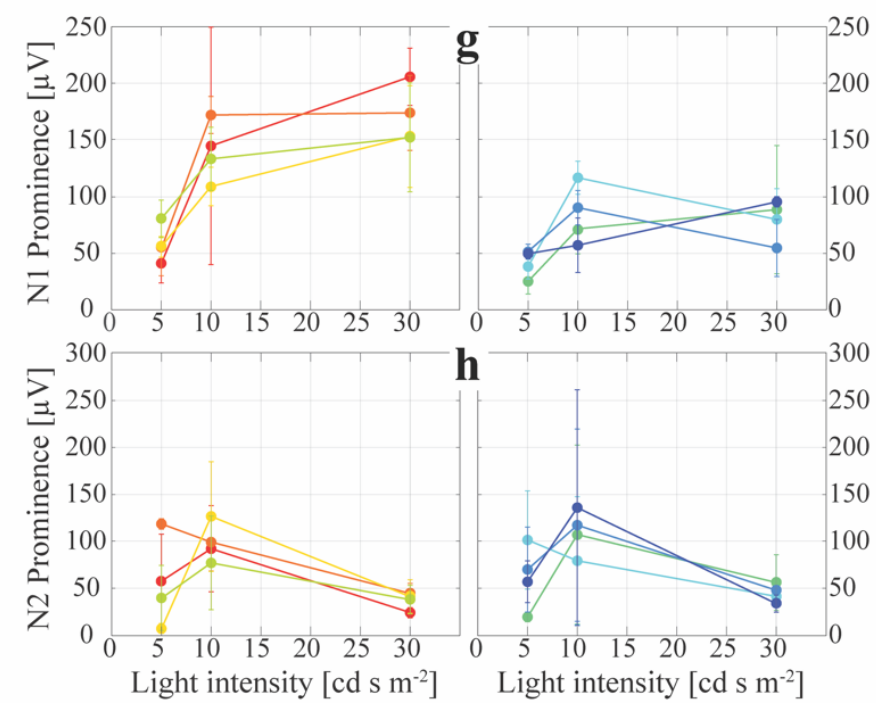

Figure 6. Evolution of field potential peaks while varying the light intensity. (a)-(d) Examples of VEPs recorded at the eight depths for different light intensities of $1,5,10$, and $30 \mathrm{~cd} \mathrm{~s} \mathrm{~m}^{-2}$, respectively (each trace is an average of $\mathrm{n}=10$ repetitions). The vertical black lines are the occurrence of the flash. Mean ( \pm s.e.m., $N=3$ animals, each with $\mathrm{n}=10$ averaged repetitions) of latencies (e)-(f) and prominences (g)-(h) of N1 and N2 peaks depending on flash intensity and cortical depth. 
a

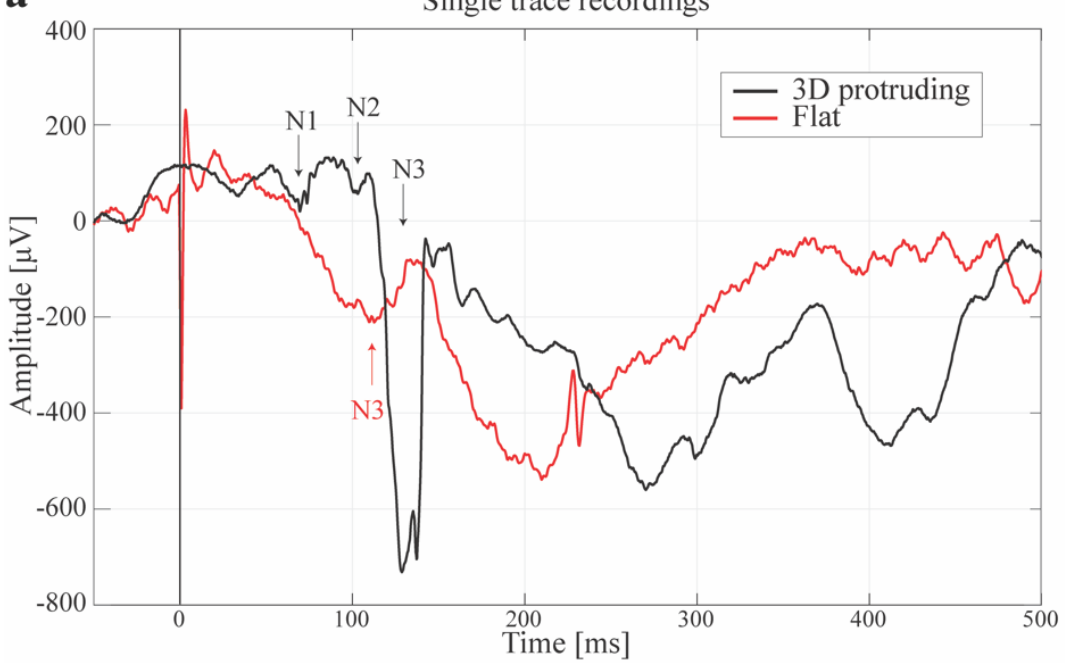

b

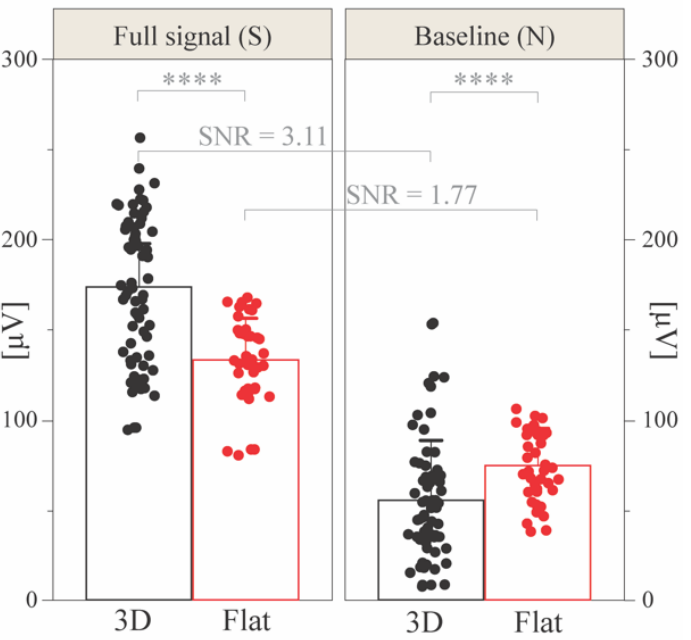

c

$3 \mathrm{~mm}$

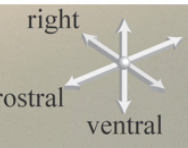

d

$50 \mu \mathrm{m}$

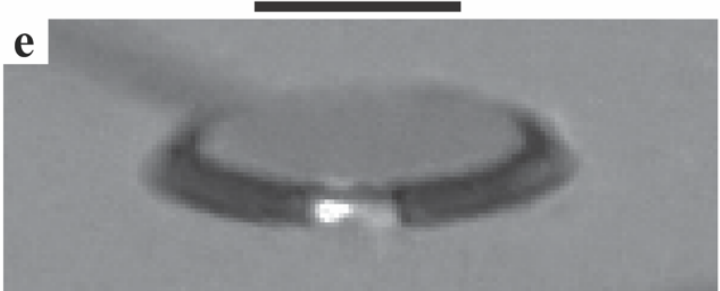

Figure 7. Signal quality and in-brain visualization of the 3D protruding electrodes. (a) Example of single trace recordings with a light intensity of $30 \mathrm{~cd} \mathrm{~s} \mathrm{~m}^{-2}$ of 3D protruding (black) and flat (red) electrodes. The vertical black line is the occurrence of the flash. (b) Signal quality evaluation by means of s.d. (mean \pm s.d.) of the traces for the full signal (F) (Mann-Whitney test, $p<$ $0.0001)$ and for the baseline (B) (Mann-Whitney test, $\mathrm{p}<0.0001)$ of 3D protruding electrodes $(\mathrm{n}=72$ recordings obtained from $\mathrm{w}=8$ electrodes and $\mathrm{N}=1$ animal) and flat electrodes $(\mathrm{n}=40$ recordings obtained from $\mathrm{w}=4$ electrodes and $\mathrm{N}=1$ animal). All the recordings were taken with a light intensity of $30 \mathrm{~cd} \mathrm{~s} \mathrm{~m}^{-2}$. (c) Photograph of a clarified mouse brain with two probes implanted. (d) Light sheet micrograph of a 3D protruding electrode within the clarified mouse brain. (e) Micrograph of a 3D protruding electrode prior to implantation. 